\title{
Control of Surface Shape in Nanostructure Formed with Femtosecond Laser Pulses
}

\author{
Godai MIYAJI and Kenzo MIYAZAKI \\ Advanced Laser Science Research Section, Institute of Advanced Energy, Kyoto University, \\ Gokasho, Uji, Kyoto 611-0011, Japan \\ E-mail: g-miyaji@iae.kyoto-u.ac.jp, miyazaki@iae.kyoto-u.ac.jp
}

\begin{abstract}
This paper reports the experimental results demonstrating that the nanostructured surface of diamondlike carbon film can be shaped so as to have a sawlike pattern with obliquely incident $p$ polarized femtosecond laser pulses. The nanoscale surface shape was observed as functions of incident angle, superimposed number and fluence of laser pulses and characterized with height and slope angle of the inclined surface. This results show that the inclined shape is formed with the non-uniform spatial distribution of local field enhanced on the nanostructured surface.

DOI: 10.2961 jlmn.2010.01.0018
\end{abstract}

Keywords: Femtosecond laser ablation, nanostructuring, local field, diamondlike carbon

\section{Introduction}

There has been considerable interest in the formation of periodic nanostructures on solid surfaces irradiated with femtosecond (fs) laser pulses, since the structure size observed so far is much smaller than the laser wavelength to suggest potential applications of fs lasers to nanoprocessing [1-4]. The characteristic properties of nanostructuring have been investigated for a variety of materials as functions of laser parameters such as polarization, fluence $F$, wavelength $\lambda$, and number of pulses $N$, where nanostructuring is usually observed with superimposed laser pulses at moderate fluences around the ablation threshold of each material [5-10]. Much attention has been focused on the physical process to understand this fascinating phenomenon [1,2]. In a series of experimental studies, the present authors have shown that local fields are enhanced to initiate nanoscale ablation on the surface having high curvatures of nanometer size, and then the nanoscale periodicity should be due to the excitation of surface plasmon polaritons (SPPs) in the surface layer [2,3]. This local field and SPP model have been shown to reconcile with the observed properties of nanostructuring.

Based on the model of nanostructuring, one can expect to shape the nanostructured surface, which may be done by means of changing the distribution of local field on the surface. In this paper, we report that the nanostructured surface of diamondlike carbon film (DLC) can be inclined to have a sawlike shape with obliquely incident fs laser pulses. The inclined surface is formed with $p$-polarized fs laser pulses, and its shape is sensitively modified with $N$ and $F$. The results show that the inclined surface is certainly formed with the non-uniform local field distribution created on the target surface in nanoscale.

\section{Experimental}

The experimental apparatus and configuration was almost the same as in our previous studies [2,3]. Briefly, we used 800 -nm, 100-fs pulses from a Ti:sapphire laser system operated at a repetition rate of $10 \mathrm{~Hz}$. Propagating through a pair of polarizer and half-wave plate, the laser pulse was focused in air at the incident angle of $\theta=0-60^{\circ}$ on the film surface with a spherical lens of $1000-\mathrm{mm}$ focal length. At the normal incidence, the focal spot size on the target was $200 \mu \mathrm{m}$ in radius with the lowest-order Gaussian intensity distribution, which was monitored with a CCD camera. The incident angle was changed by a precision rotation stage holding the target. With an increase in $\theta$, the fluence $F$ decreases by a factor $\cos \theta$, and then the pulse energy was increased to maintain a constant value of $F$ on the target. The pulse energy used was $130-350 \mu \mathrm{J}$ for $F=$ $60-140 \mathrm{~mJ} / \mathrm{cm}^{2}$ at different values of $\theta$. The superimposed number of laser pulses $N$ on the target was in a range of $N=1-1000$.

The target used was DLC film of $500 \mathrm{~nm}$ in thickness, deposited on polished silicon substrates with a plasmabased ion implantation system. The surface roughness was measured to be less than $1 \mathrm{~nm}$ with a scanning probe microscope (SPM) using a micro cantilever with a tip apex of $15 \mathrm{~nm}$ in radius. The surface morphology was observed with a scanning electron microscope (SEM) and the SPM. The periodic nanostructure on the SEM and SPM images was analyzed with the two-dimensional Fourier transform.

\section{Results and discussion}

Figure 1 shows the SEM images and their lateral scans of the DLC target surface irradiated at $\theta=0^{\circ}, 20^{\circ}, 40^{\circ}$ and $60^{\circ}$ with $N=100$ of $p$-polarized laser pulses at $F=120$ $\mathrm{mJ} / \mathrm{cm}^{2}$. At $\theta=0^{\circ}$, the periodic structure is formed with the mean period size of $d \sim 100 \mathrm{~nm}$ on the film surface, where the linelike grooves are highly oriented to the direction perpendicular to the laser polarization. For $\theta=20^{\circ}$, the nanostructured surface with $d \sim 110 \mathrm{~nm}$ starts to have a slope along the direction of wave vector $\boldsymbol{k}$ of the incident field $\boldsymbol{E}$. The slope angle $\Phi$ and height $h$ were measured to be $\Phi \sim 10^{\circ}$ and $h \sim 10 \mathrm{~nm}$, where $\Phi$ and $h$ are defined as the inset in Fig. 1(b). For a larger incident angle of $\theta=40^{\circ}$, the nanostructured surface with $d \sim 120 \mathrm{~nm}$ is sloped to have $\Phi \sim 30^{\circ}$ and $h \sim 60 \mathrm{~nm}$. With an increase in $\theta$ to $60^{\circ}$, the surface slope increases to have $h \sim 110 \mathrm{~nm}$ with $\Phi \sim$ 

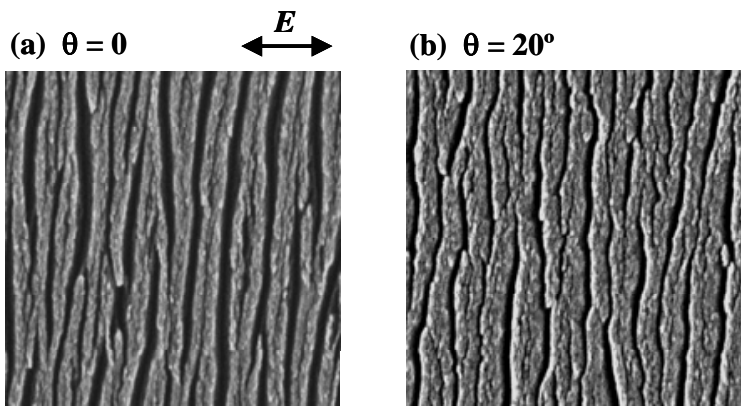

(c) $\boldsymbol{\theta}=\mathbf{4 0 ^ { \circ }}$

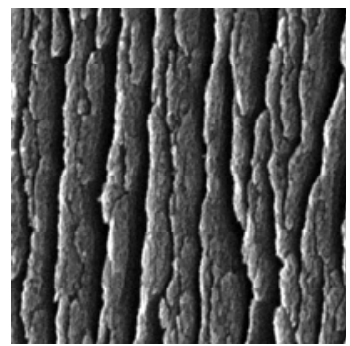

(d) $\theta=60^{\circ}$
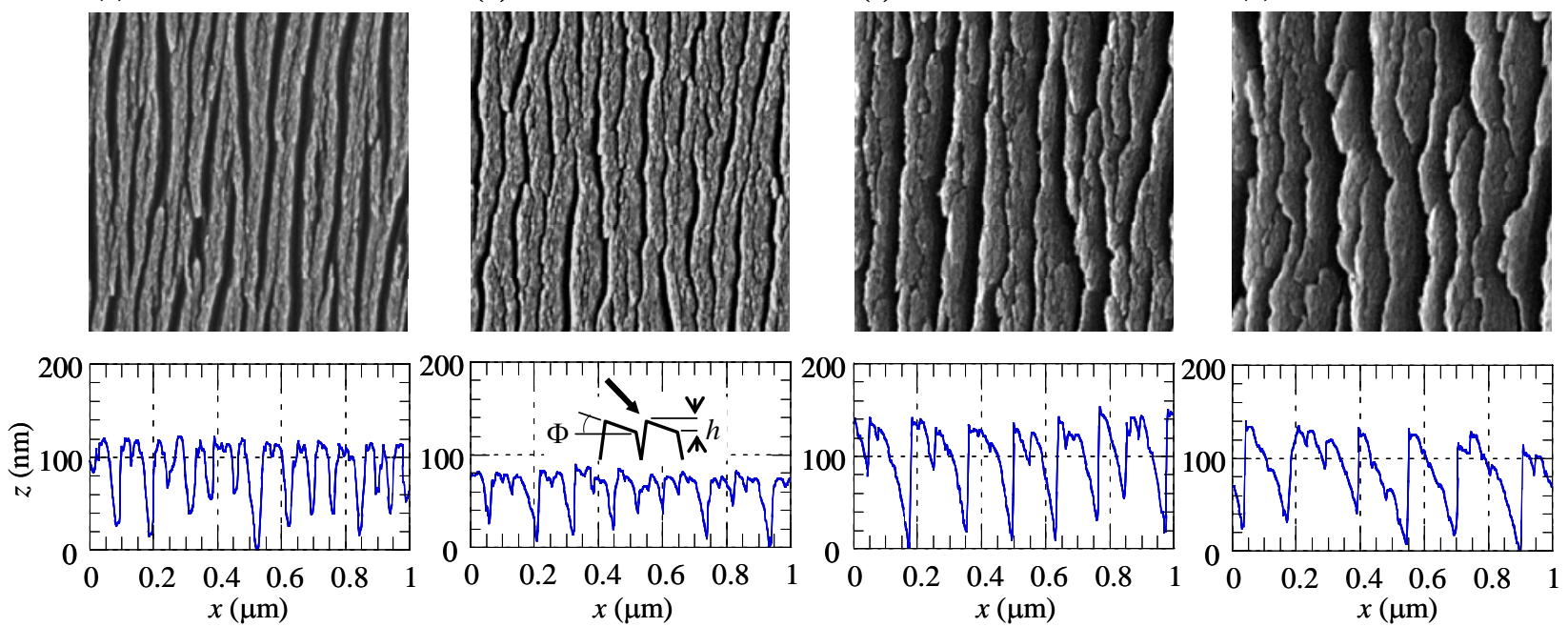

Fig. 1 SEM images and their lateral $(x-z)$ scans of the DLC surface irradiated at (a) $\theta=0^{\circ}$, (b) $\theta=20^{\circ}$, (c) $\theta=40^{\circ}$, and (d) $\theta=60^{\circ}$ with $N=100$ of $p$-polarized laser pulses at $F=120 \mathrm{~mJ} / \mathrm{cm}^{2}$. The image is for the central area of $1 \times 1$ $\mu \mathrm{m}^{2}$ on the focal spot. The scan is along the polarization $(x)$ direction denoted by the arrow $\boldsymbol{E}$, and the ordinate of $z=0$ indicates the lowest $z$ position in the observed area with the SPM. The inset in (b) defines the height $h$ and the angle $\Phi$ of surface slope, and the arrow denotes the incident direction of laser pulses.

$40^{\circ}$ at $d \sim 180 \mathrm{~nm}$. It is noted in Fig. 1(d) that the inclined surface straightly goes down to the bottom of the neighboring groove to form a typical sawlike shape. We have confirmed that $h$ and $\Phi$ increase monotonously with increasing $\theta$ at fixed values of $F$ and $N$. These results strongly suggest that the obliquely incident $E$-field produces the periodically enhanced non-uniform local field to form the sawlike ablation trace.

The inclined surface can be formed with only $p$ polarization of fs laser pulses, and never with s-polarization.
To confirm this, the experiment was made with s-polarized pulses under the same conditions as in Fig. 1. The SEM images observed at $\theta=20^{\circ}, 40^{\circ}$ and $60^{\circ}$ are shown in Fig. 2, together with their vertical scans. Note that no inclined surface is formed in the nanostructure with $d=60-80 \mathrm{~nm}$. The nanostructure in Fig. 2(c) is very shallow, in contrast to those observed with $p$-polarization. This is due to the increased reflection of the s-polarized laser pulse at the large incident angle $\theta=60^{\circ}$, resulting in a decrease in the effective value of $F$ on the surface. The polarization-dependent

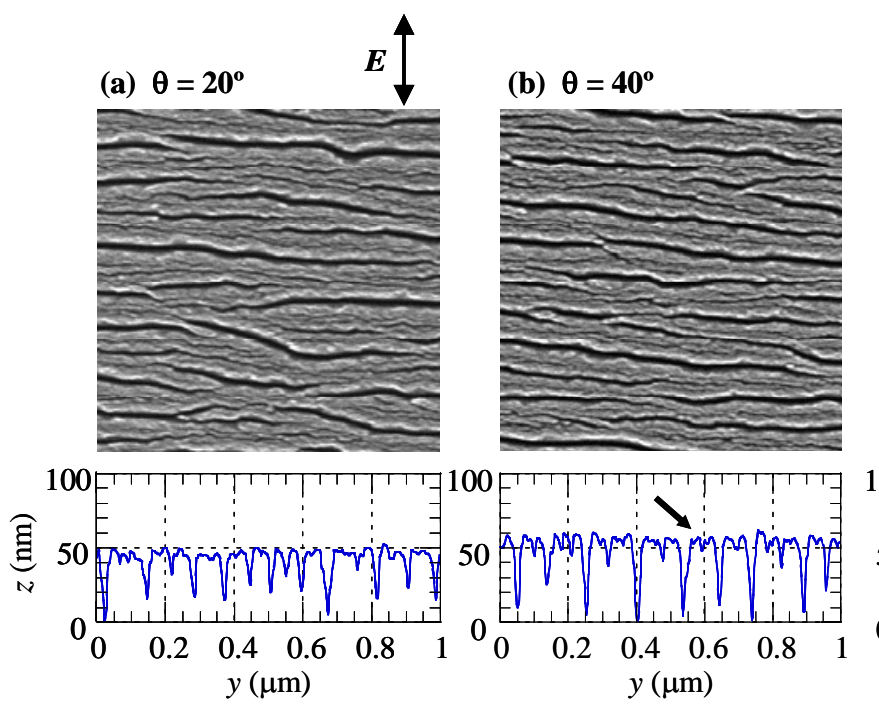

(c) $\theta=60^{\circ}$

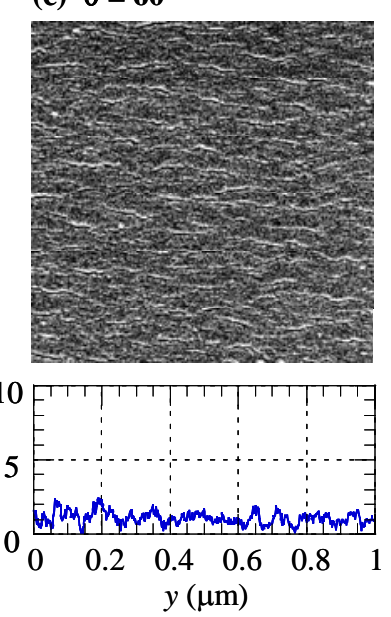

Fig. 2 SEM images and their vertical (y-z) scans of the DLC surface irradiated at (a) $\theta=20^{\circ}$, (b) $\theta=40^{\circ}$ and (c) $\theta=60^{\circ}$ with $N=100$ of s-polarized laser pulses at $F=120 \mathrm{~mJ} / \mathrm{cm}^{2}$. The image is for the central area of $1 \times 1 \mu \mathrm{m}^{2}$ on the focal spot. The scan is along the polarization $(y)$ direction denoted by the arrow $\boldsymbol{E}$, and the ordinate of $z=0$ indicates the lowest $z$ position in the observed area with the SPM. The arrow in (b) denotes the incident direction of laser pulses. 


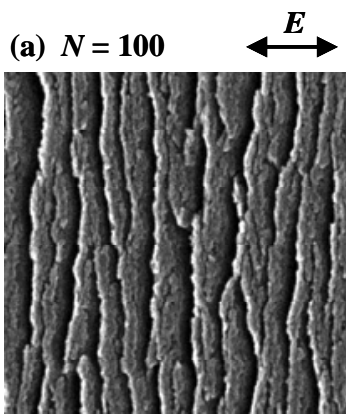

(b) $N=\mathbf{3 0 0}$

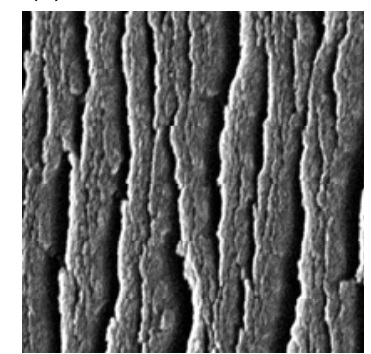

(c) $N=\mathbf{1 0 0 0}$
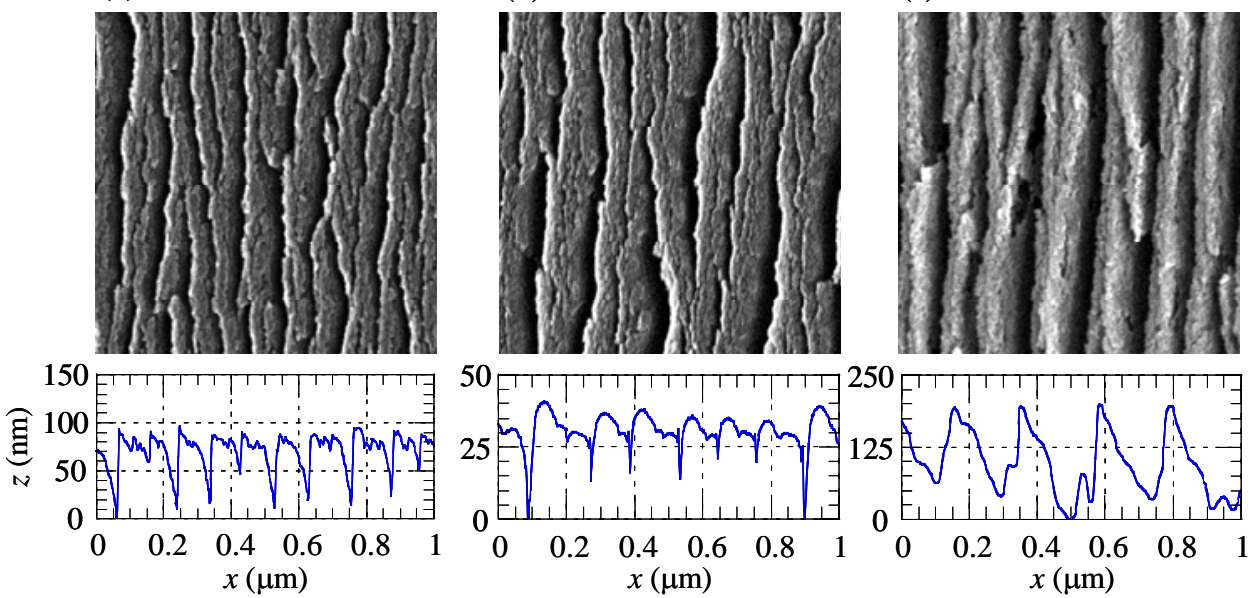

Fig. 3 SEM images and their lateral ( $x-z)$ scans of the DLC surface irradiated with (a) $N=100$, (b) $N=300$, and (c) $N=$ 1000 of $p$-polarized laser pulses at $F=100 \mathrm{~mJ} / \mathrm{cm}^{2}$ and $\theta=40^{\circ}$. The image area, the scan direction, and the incident direction of laser pulses are the same as in Fig.1.

shapes observed provide an experimental evidence that the local field enhanced on the surface initiates the nanoscale ablation on the surface with high curvature [3].

The superimposed number of laser pulses $N$ should be an important parameter to manipulate the nanostructured surface shape, since $N$ is well known to strongly affect on the nanostructure formation [2-4]. We observed the nanostructured surface shape as a function of $N$ of $p$-polarized pulses at fixed values of $F$ and $\theta$. Figure 3 shows the SEM images of DLC surface observed with $N=100,300$ and 1000 at $F=100 \mathrm{~mJ} / \mathrm{cm}^{2}$ and $\theta=40^{\circ}$. For $N=100$, the nanostructured surface with $d \sim 100 \mathrm{~nm}$ is observed to have a gentle slope of $h \sim 10 \mathrm{~nm}$ with $\Phi \sim 10^{\circ}$. It is noted that an increase in $N$ to $N=300$ and 1000 makes the surface slope steeper to $\Phi \sim 15^{\circ}$ with $h \sim 15 \mathrm{~nm}$ at $N=300$ and to $\Phi \sim 50^{\circ}$ with $h \sim 170 \mathrm{~nm}$ at $N=1000$, respectively. The surface slope $\Phi$ observed for $N=1000$ at $\theta=40^{\circ}$ is larger than that for $\theta=60^{\circ}$ in Fig. 1(d). The $N$-dependent surface shapes observed are consistent with the above conclusion that the non-uniform local field is produced to form a slope of the periodically nanostructured surface.

Since an increase in $F$ is expected to provide almost the same effect on nanostructuring as an increase in $N$, the surface morphology was observed as a function of $F$ at fixed values of $N$ and $\theta$. The results are shown in Fig. 4. As expected, the slope increases as $\Phi \sim 10^{\circ}, 40^{\circ}$ and $50^{\circ}$ for $F$ $=60,80$ and $140 \mathrm{~mJ} / \mathrm{cm}^{2}$, respectively, together with the increasing period of the nanostructure. The results obtained show that the surface shape is more sensitive to $F$ than to $N$, whereas both parameters play a similar role in nanostructuring. This is consistent with the fact that the ablation itself is a phenomenon with a sharp threshold of $F$.

To see the generation of non-uniform local-fields with obliquely incident laser pulses, we calculated near-field distributions on nanostructured surfaces, using a finitedifference time-domain (FDTD) method [11]. We consider

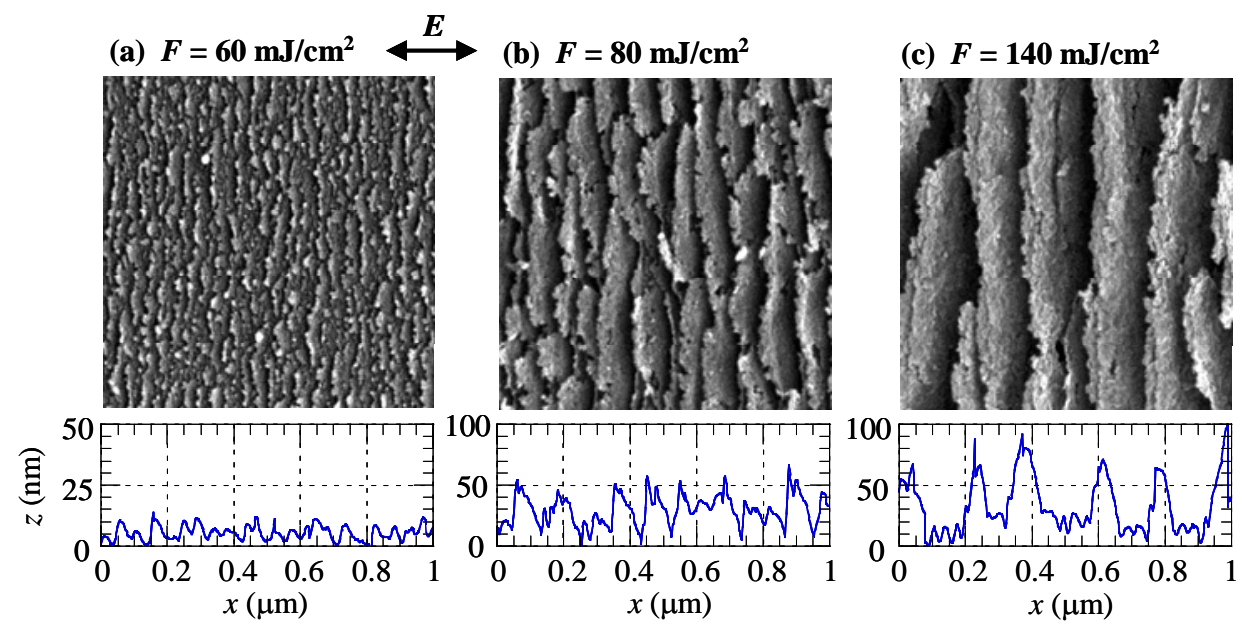

Fig. 4 SEM images and their lateral $(x-z)$ scans of the DLC surface irradiated with $N=1000$ of $p$-polarized laser pulses at (a) $F=60 \mathrm{~mJ} / \mathrm{cm}^{2}$, (b) $F=80 \mathrm{~mJ} / \mathrm{cm}^{2}$, and (c) $F=140 \mathrm{~mJ} / \mathrm{cm}^{2}$ for $\theta=60^{\circ}$. The image area, the scan direction, and the incident direction of laser pulses are the same as in Fig. 1. 
(a)

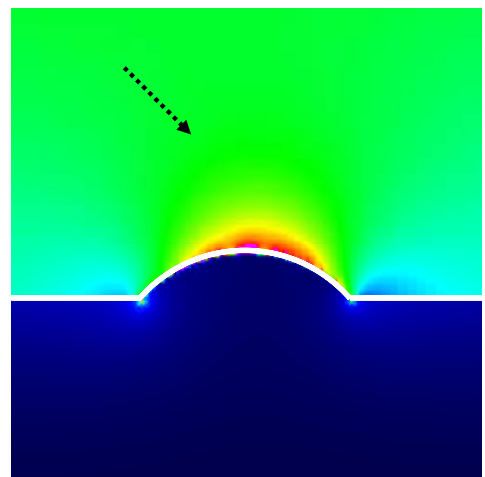

(b)

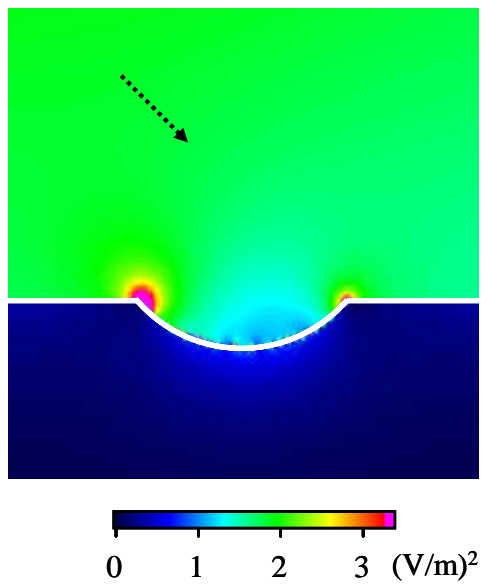

Fig. 5 Calculated field intensity distribution around (a) a hill and (b) a valley on the DLC surface irradiated with $p$-polarized light incident at $\theta=40^{\circ}$. The hill and valley are assumed to have the radius of $30 \mathrm{~nm}$ with the height and depth of 10 $\mathrm{nm}$. The arrows denote the incident direction of laser light.

two kinds of model surface of nanometer size. Those include (a) a hill of $10 \mathrm{~nm}$ in height and (b) a valley of $10 \mathrm{~nm}$ in depth, both of which is $30 \mathrm{~nm}$ in radius, as illustrated in Fig. 5. The $p$-polarized $800-\mathrm{nm}$ laser field of $E=1 \mathrm{~V} / \mathrm{m}$ is incident at $\theta=40^{\circ}$ from air (dielectric constant $\varepsilon_{1}=1$ ) to these surfaces. Based on our experimental results [2], the free electron density of $N_{\mathrm{e}} \sim 2 \times 10^{22} \mathrm{~cm}^{-3}$ is assumed for the surface layer concerned. Taking into account a simple Drude model with $N_{\mathrm{e}}$, we used $\varepsilon_{2}=-8.72+i 3.18$ as the dielectric constant of GC layer to be ablated. Figure 5 shows the calculated results of the time-averaged field intensity on the surfaces. Note that the non-uniform local field is generated with its peak intensity shifted to the right on the hill, whereas the enhanced field is localized at the left edge of the valley. The non-uniform distributions of local field should produce the slope of nanostructured surface in ablation. The calculation has represented that spolarized pulses never produces such non-uniform field distribution as in Fig. 5.

\section{Conclusions}

We have shown that the nanostructured DLC film surface can be modified to have a sawlike shape with obliquely incident $p$-polarized fs laser pulses, and the shape is also sensitive to the superimposed number of laser pulses and the laser fluence. The results demonstrate that the inclined surface is formed with the non-uniform spatial distribution of local field enhanced on the surface roughness.

\section{Acknowledgments}

The authors thank Y. Miyatani and K. Zhang for their help in the experiment. This work was partially supported by the Grant-in-Aid for Scientific Research (A)18206010, Grant-in-Aid for Young Scientists (B) 20760033, and a research granted from the Murata Science Foundation A81158.

\section{References}

[1] K. Miyazaki: "Nanophotonics and Nanofabrication” ed. by M. Ohtsu (Wiley-VCH, Weinheim, 2009), Chap.10.

[2] G. Miyaji, K. Miyazaki: Opt. Express 16, 1626516271 (2008).

[3] G. Miyaji, K. Miyazaki: Appl. Phys. Lett. 91, 123102 (2007).

[4] G. Miyaji, K. Miyazaki: Appl. Phys. Lett. 89, 191902 (2006).

[5] N. Yasumaru, K. Miyazaki, J. Kiuchi: Appl. Phys. A 76, 983-985 (2003).

[6] N. Yasumaru, K. Miyazaki, J. Kiuchi: Appl. Phys. A 79, 425-427 (2004).

[7] N. Yasumaru, K. Miyazaki, J. Kiuchi: Appl. Phys. A 81, 933-937 (2005).

[8] K. Miyazaki, N. Maekawa, W. Kobayashi, N. Yasumaru, J. Kiuchi: Appl. Phys. A 80, 17-21 (2005).

[9] Q. Z. Zhao, S. Malzer, L. J. Wang: Opt. Lett. 32, 19321934 (2007).

[10] J. Reif, O. Varlamova, F. Costache: Appl. Phys. A 92, 1019-1024 (2008).

[11] A. Taflove, S. C. Hagness: “Computational electrodynamics: the finite-difference time-domain method, 3rd ed.”, (Artech House, Boston, 2005), Chap. 2 and 3.

(Received: July 9, 2009, Accepted: February 21, 2010) 